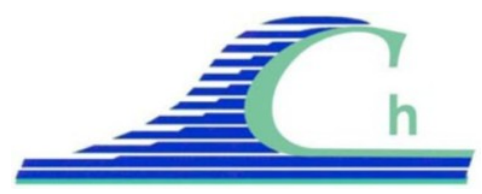

XII ${ }^{\text {ìmes }}$ Journées Nationales Génie Côtier - Génie Civil

Cherbourg, 12-14 juin 2012

DOI:10.5150/jngcgc.2012.028-E（C) Editions Paralia CFL

disponible en ligne - http://www.paralia.fr - available online

\title{
Evolution à moyen terme du contexte hydrodynamique et morphosédimentaire la baie d'Essaouira (Maroc atlantique)
}

\author{
Abdelhadi EL MIMOUNI ${ }^{1}$, Lahcen DAOUDI $^{1}$ \\ 1. Laboratoire de Géosciences et Environnement, Département des Sciences de la Terre, \\ Faculté des Sciences et Techniques de Marrakech, BP. 549, Maroc. \\ abdel_elmimouni@yahoo.fr
}

\section{Résumé :}

La caractérisation de la dynamique morphosédimentaire et les facteurs y agissant dans la baie d'Essaouira a nécessité plusieurs campagnes de mesures hydrodynamiques, bathymétriques et sédimentaires, qui ont fait appel à l'utilisation de plusieurs techniques de mesure. Les enregistrements obtenus montrent que l'influence de la digue du port d'Essaouira et surtout de l'île de Mogador sur la propagation de la houle est remarquable. Cette situation est à l'origine de la naissance de plusieurs types de courants plus au moins importants dans la baie. La comparaison des données obtenues avec celle d'une étude similaire réalisée en 1955 montre une variation très nette dans la répartition des sédiments au fond de la baie, ce qui dénote l'évolution des conditions hydrodynamiques et morphologique au niveau de la baie.

\section{Mots-clés :}

Littoral - Hydrodynamisme - Bathymétrie - Granulométrie - Evolution - Baie d'Essaouira - Maroc

\section{Introduction}

Situé sur la façade atlantique du Maroc, le littoral d'Essaouira qui s'étend sur plusieurs dizaines de kilomètres présente une grande variété morphologique: estuaire, baie, lagunes, plages, flèches sableuses, falaises, estrans rocheux, ... . (WEISROCK, 1980 ; SIMON, 2000 ; LHARTI et al., 2006). Depuis l'antiquité, ce littoral est le siège d'une dynamique sableuse très active en relation avec des conditions climatiques et hydrodynamiques très sévères (HANDER, 1993). Cette dynamique sableuse s'est fortement accentuée dès le début des années 1990, suite à une relance de divers secteurs socio-économiques associée à une dégradation accentuée de l'environnement (extension de la ville aux dépens des édifices dunaires, installations des infrastructures touristiques, déforestation intensive, surexploitation de la nappe phréatique, extraction des sables et des granulats sur la plage,...), (GENTILE, 1997 ; EL MIMOUNI, 2009).

La baie d'Essaouira délimitée par la digue du port de la ville d'Essaouira au nord, par l'estuaire de l'oued Ksob au sud et par l'île de Mogador à l'ouest, constitue une des zones les plus complexes de ce littoral. La dynamique morphosédimentaire de la baie d’Essaouira, a été très peu étudiée ; une seule une étude granulométrique a été effectuée, 
au niveau de la baie, en 1955 par le Laboratoire d'Hydraulique Neyrpic. Les rares autres travaux qui ont été effectués concernent la morphologie générale des dunes sableuses dans la partie haute de la plage et sur le continent (GENTILE, 1997 ; LHARTI et al., 2006 ; SIMON, 2000).

Cet article vise à une meilleure connaissance des processus mis en œuvre dans la dynamique spatio-temporelle des dépôts sédimentaires et des changements morphodynamiques au niveau de la baie d'Essaouira, en se basant sur les résultats de plusieurs campagnes de mesures hydrodynamiques, bathymétriques et sédimentaires qui nous avons effectuées (voir plus bas).

\section{Matériels et méthodes}

La caractérisation et simulation des transports sédimentaires dans la baie dEssaouira, qui mènent à la bonne connaissance de la dynamique morphosédimentaire et les facteurs y agissant, a nécessité plusieurs campagnes de mesures hydrodynamiques, bathymétriques et sédimentaires, qui font appel à l’utilisation de plusieurs techniques de mesure.

L'étude courantométrique a nécessité l'utilisation d'un appareil de mesure de type ADCP (Acoustic Doppler Current Profiler), cet appareil a été installé sur des secteurs représentatifs (avec de différentes spécificités hydrodynamique) tout au long de la baie d'Essaouira. Trois campagnes de mesure ont été effectuées sous des conditions climatiques et hydrodynamiques variables ; la première en juin 2005, la seconde en mai 2006 et la dernière en Septembre 2006 (voir figure 1). Pour chaque station de mesure, on détermine les paramètres concernant à la fois les courants (directions générales, vitesse transversale, vitesse longitudinale et vitesse moyenne) et la houle (direction générale, hauteur et période des pics).

L'étude bathymétrique de la baie est réalisée en utilisant un échosondeur Singlebeam couplé à un positionnement par satellite de type GPS différentiel, dont la précision atteinte est de l'ordre de 1 mètre en $\mathrm{X}, \mathrm{Y}$ et $1 \mathrm{~cm}$ en $\mathrm{Z}$. Au total, 279 points de sonde couvrant toute la supérficie de la baie ont été effectués et une soixantaine d'échantillons prélevées (voir figure 1).

Le traitement granulométrique des échantillons prélevés a été fait par un granulométre laser de type Coulter. Les données granulométriques obtenues ont été traitées à l'aide d'un logiciel intégré au microgranulomètre LS 230. Les paramètres habituels (taille moyenne $(\mu)$, classement $(\sigma)$ et asymétrie $(S k)$ ) (FOLK \& WARD, 1957), ont été déterminés en adoptant la méthode des moments statistiques (RIVIERE, 1977). 


\section{XII ${ }^{\text {èmes }}$ Journées Nationales Génie Côtier - Génie Civil \\ Cherbourg, 12-14 juin 2012}

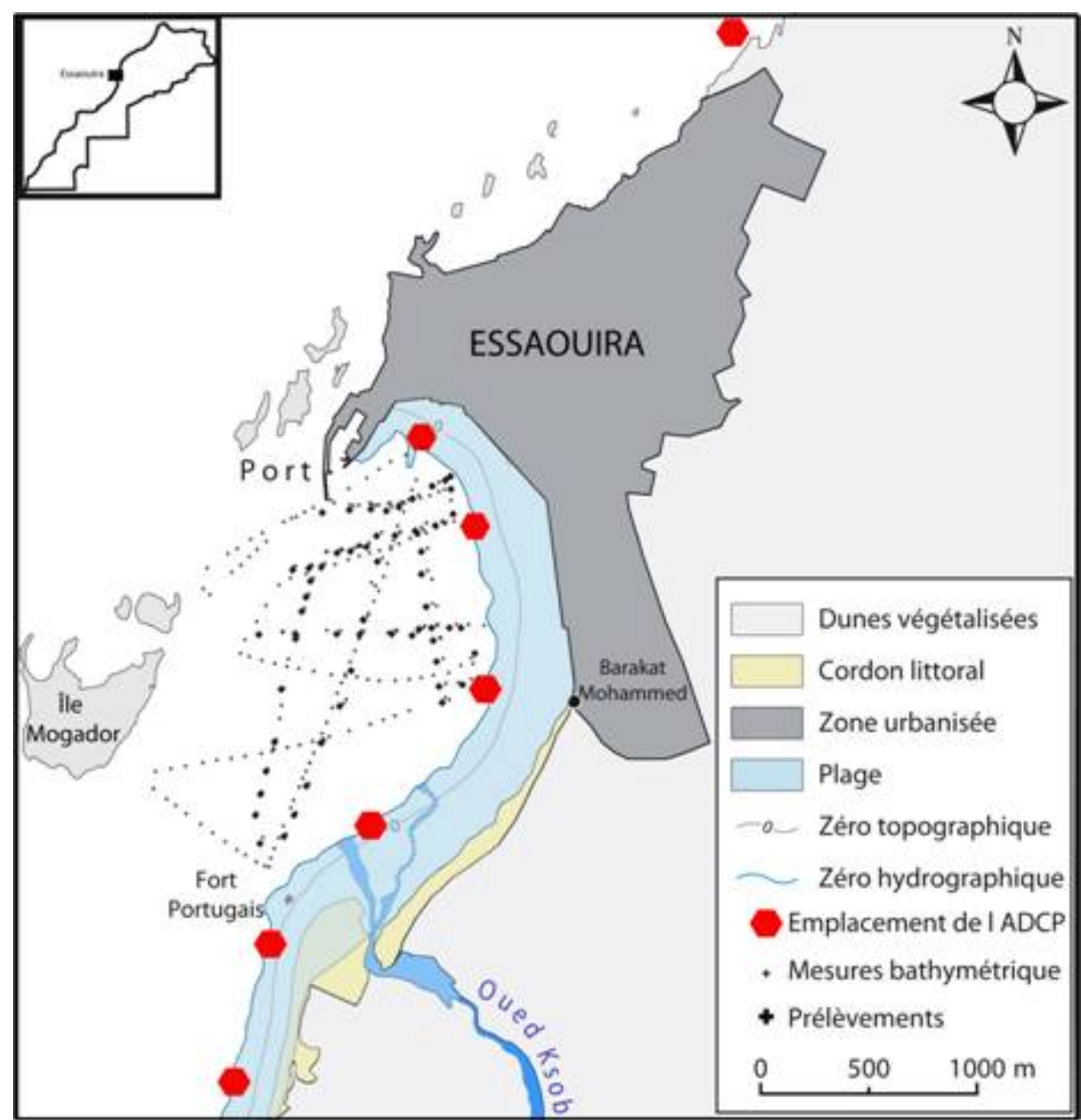

Figure 1. Carte de localisation du secteur d'étude avec emplacement des courantomètres et de la zone de prélèvement des échantillons.

\section{Résultats obtenus et Discussion}

\subsection{Analyse hydrodynamique}

Les phases hydrodynamiques identifiées pour toutes les campagnes de mesures montrent clairement que la variabilité des conditions est étroitement liée à la météorologie, notamment lors des périodes de forte agitation (REICHMÜTH, 2003 ; SEDRATI 2006 ; REICHMÜTH \& ANTHONY, 2007; SEDRATI \& ANTHONY, 2007). Ainsi, la première campagne de mesures hydrodynamique effectuées (juin 2005) dans la baie d'Essaouira coïncide avec un "temps calme" marqué par une quasi absence de la houle durant toute la période de mesures, les rares vagues enregistrées n’étant pas exploitables contrairement aux deux autres campagnes (mai et septembre 2006) où le temps a été relativement agité. Les résultats obtenus lors des différentes campagnes de mesures hydrodynamiques couplées aux situations météorologiques de la plage d’Essaouira mettent en évidence une variabilité spatiotemporelle. 


\subsubsection{La houle}

$\mathrm{Au}$ nord et au sud de la baie, les analyses spectrales de la houle ont démontré la présence d'une houle infragravitaire, liée principalement à l'ouverture de ces deux sites mais surtout à une morphologie d'avant-plage et de plage complexe, marquée, pour le nord par des secteurs à rochers variablement denses, et pour le sud à l'influence très probable du prisme de sables éoliens et estuariens pris en charge par la dérive littorale importante vers le sud qui s'exerce sur le littoral marocain atlantique.

Dans la baie, les enregistrements montrent la dominance, plus classique, de la houle incidente de gravité. L'influence de la digue du port d'Essaouira et surtout de l'île de Mogador sur la propagation de la houle est très remarquable. La houle incidente qui est habituellement de direction NW pénètre par le couloir entre l'île de Mogador et le port d'Essaouira et prend des directions divergentes, multidirectionnelle par diffraction. À l'extrême nord de la plage, près du port, la houle prend plusieurs directions : SW par sa diffraction sur les deux jetées du port, NW liée à la réflexion sur la digue de la plage, et une troisième direction NE résultant de la réfraction de la houle sur l'avant-côte. Au centre de la baie et au niveau de l'estuaire, la direction de la houle fluctue entre $\mathrm{W}$ et NNW (figure 2).

\subsubsection{Les courants}

Au nord de la ville d'Essaouira et à l'extrême sud de la zone d'étude, le même type de courant a été enregistré ; c'est un courant fort de direction SW qui correspond vraisemblablement au courant de dérive littorale. Au niveau de la baie, aussi bien en condition de faible que de forte agitation, la divergence de la houle entraîne la naissance de plusieurs types de courants. Le premier type de direction $\mathrm{N}$ à NNW et le second de direction SSW à SW sont liés à l'orientation de la plage et à la morphologie générale de la ligne de rivage. Le troisième type de courant est enregistré au niveau de l'estuaire ; il est multidirectionnel et est lié à l'interaction de l'hydrodynamisme marin et fluvial (figure 2).

\subsection{Analyse morphosédimentaire}

Du point de vue morphologique, les levés bathymétriques effectués montrent l'existence d'un couloir marin de direction SE entre l'île de Mogador et la digue du port d'Essaouira où des profondeurs de $-13 \mathrm{~m}$ ont été levées. Les profondeurs enregistrées au nord de ce couloir près du port d'Essaouira ( 0 à $-2,5 \mathrm{~m}$ ) et vers les sud au niveau du fort portugais ( 0 à $-5 \mathrm{~m}$ ), révèlent un exhaussement global des fonds.

Du point de vue sédimentologique, suite à l'analyse granulométrique des échantillons prélevés dans la baie d'Essaouira, une carte de répartition des sédiments a été établie (figure 3A). Selon cette carte, la vase occupe un large domaine au centre de la baie correspondant au couloir compris entre l’île de Mogador et la jetée du port. Cette zone 


\section{XII ${ }^{\text {èmes }}$ Journées Nationales Génie Côtier - Génie Civil \\ Cherbourg, 12-14 juin 2012}

occupée par de la vase est entourée par des sables très fins et des sables fins qui se dispersent tout le long de la zone infratidale de la plage. Les sables moyens sont limités à la zone de la plage située au niveau de l'estuaire de Ksob, alors que la zone des galets est plaquée contre l'île de Mogador et la jetée du port.

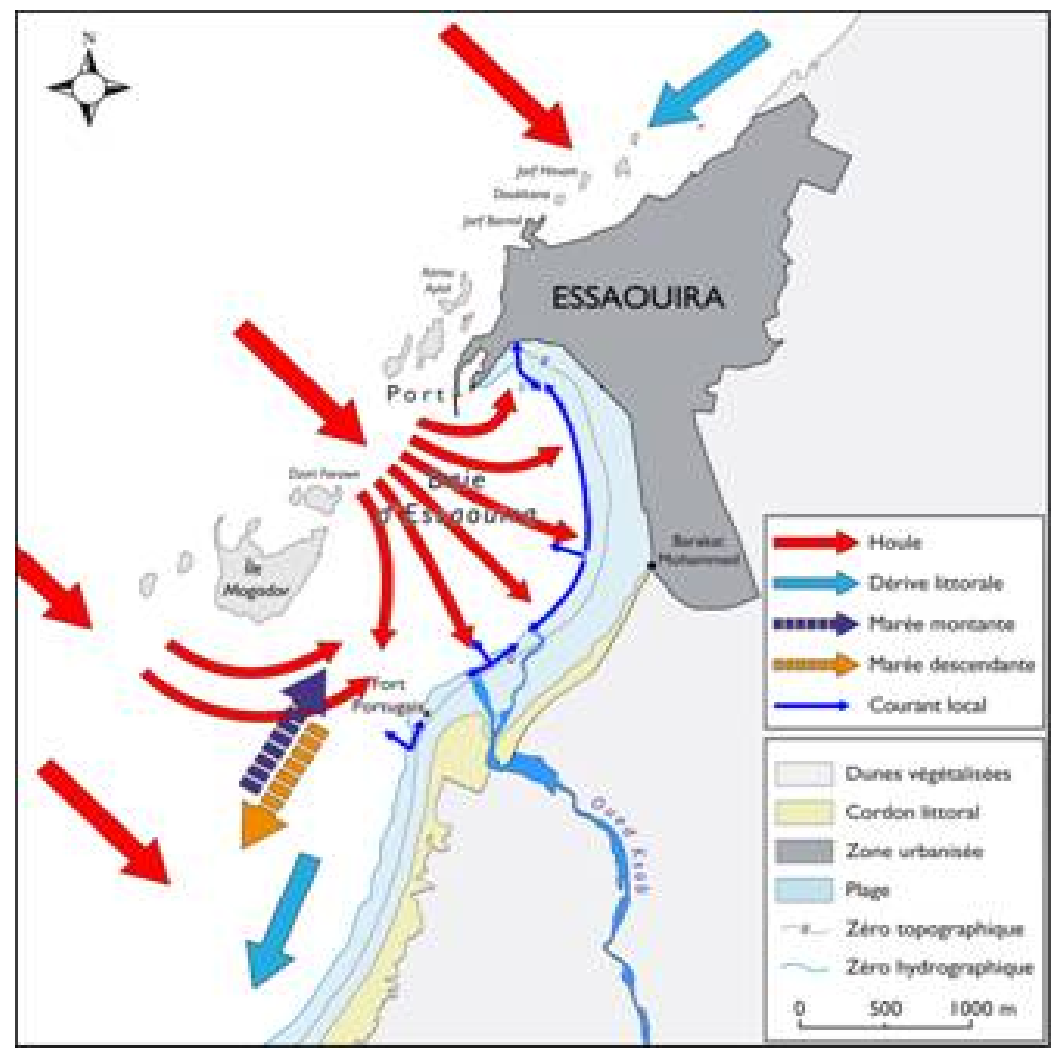

Figure 2. Synthèse schématique de la circulation hydrodynamique dans le secteur d'Essaouira.

L'analyse sédimentologique de cette répartition montre que d'une façon générale, la distribution des sédiments superficiels dans la baie d'Essaouira montre deux secteurs différenciés par leur caractéristiques sédimentologiques: le centre de la baie qui correspond à sa partie la plus profonde (-6 à -13 m) et qui se caractérise par l'abondance de sédiments fins (vase et sables fins) matérialisant un milieu de faible énergie ; la zone infratidale de la plage ( 0 à $-5 \mathrm{~m}$ de profondeur) et la zone de l'estuaire où les courants de marée et les courants locaux induisent une agitation du milieu et le dépôt de sédiments plus grossiers.

La comparaison des résultats sédimentologiques obtenus avec ceux d'une étude similaire réalisée en 1955 par le Laboratoire d’Hydraulique Neyrpic (figure 3B) montre la même tendance évolutive de distribution des sédiments. En effet, la carte de la figure 3B montre une concentration de la vase et des sables très fins au centre de la baie, les sables moyens se localisant le long de la partie infratidale de la plage d'Essaouira tandis 


\section{Thème 2 - Dynamique sédimentaire}

que les sables grossiers et les galets s'étendent vers le fort portugais et le sud de la baie. Cependant, la zone occupée par la vase est moins étendue que dans le premier cas, elle se limite à un couloir étroit juxtaposé à la jetée du port. Par ailleurs, les sédiments grossiers (sables grossiers et galets) occupent un domaine beaucoup plus large.
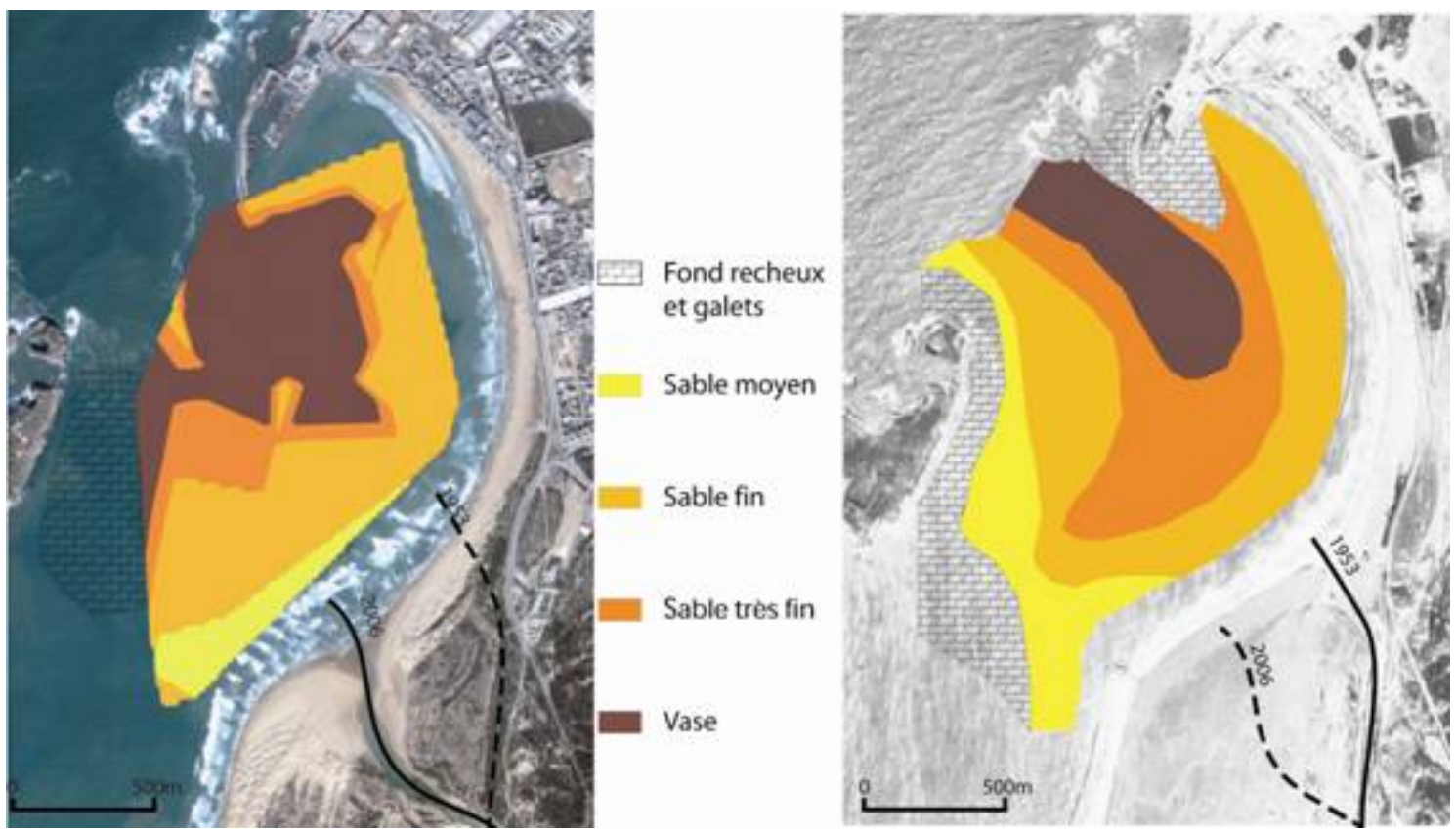

Figure 3. Répartition des sédiments dans la baie d’Essaouira : A) actuel, B) en 1955.

En absence d'études courantométriques antérieures, on peut avancer que la comparaison de ces deux cartes plaide, d'une façon générale, en faveur d'une variation des conditions hydrodynamiques au niveau de la baie d'Essaouira. En effet, la répartition des sédiments suggère que du point de vue hydrodynamique, la baie est actuellement mois soumise à l'agitation qu'en 1955. Cette situation est vraisemblablement en relation avec les travaux d'aménagement que le port a connu et qui concernent l'extension de la digue en 1963, ce qui a permis la création de condition plus calmes dans la baie. Par ailleurs, nous avons pu montrer à travers l'étude des photos aériennes (EL MIMOUNI et al., 2010) que durant le siècle dernier, vers la partie avale de l'oued Ksob, le lit mineur fluctue entre les directions Nord et Ouest. Ces fluctuations du lit mineur représentent un déplacement de l'embouchure de l'oued d'environ $1000 \mathrm{~m}$ depuis la fin du XIX ${ }^{\text {ème }}$ siècle. La comparaison des deux photos aériennes (figure 3) montre un déplacement de l'exutoire de l'oued Ksob d'environ $500 \mathrm{~m}$ vers le sud. Ceci représente un élément clef de la dynamique morphosédimentaire de la baie d'Essaouira et qui permet d'y exercer un contrôle sur les conditions hydrodynamiques. 


\section{XII ${ }^{\text {èmes }}$ Journées Nationales Génie Côtier - Génie Civil \\ Cherbourg, 12-14 juin 2012}

\section{Conclusion}

La baie d'Essaouira constitue une zone complexe du littoral d'Essaouira soumise à des processus hydrodynamiques et sédimentaire particuliers. Ces processus varient considérablement dans le temps et dans l'espace, et semblent profondément modifiés par les actions anthropiques. La complexité des processus hydrodynamiques est principalement liée à la forme très particulière de la houle au niveau de la baie d'Essaouira. Cette particularité résulte de la déformation de l'onde incidente venant de l'Atlantique par les obstacles que représentent la jetée du port d'Essaouira et surtout l'île de Mogador. L’oued Ksob de son côté, joue un rôle primordial dans cette évolution par : (1) l'apport sédimentaire dont il est responsable en périodes de crue ; ces sédiments accumulés dans la baie d'Essaouira lors des crues sont redistribués par la suite sur la plage selon les directions générales des courants, (2) par les changements de la trajectoire de son lit mineur au niveau de l'exutoire, au cours du temps.

Comme nous l'avons déjà noté précédemment, la dynamique morphosédimentaire de la baie d'Essaouira a été très peu étudiée. En absence de telles études, nous disposons avec ce travail, d'une base de données fiable donnant des informations relativement sûres sur l'hydrodynamique des courants et de la houle, sur la morphologie et la bathymétrie de la baie et sur la répartition des sédiments superficiels. Enfin, nos travaux au cours de cette étude ont visé essentiellement la baie d'Essaouira ; l'étude hydrodynamique de cette baie ne peut être étudiée de façon isolée par rapport au reste du littoral d'Essaouira. Des efforts de recherche sont donc à faire dans ce sens, pour caractériser l'évolution hydrodynamique et morphosédimentaire de ce littoral et l'insertion de cette zone côtière dans ce contexte d'ensemble.

\section{Références bibliographiques}

EL MIMOUNI A. (2009). Approche expérimentale de suivi de la dynamique morphosédimentaire du littoral d'Essaouira, Maroc: Implication pour un aménagement raisonné. Thèse Université du Littoral Côte d’Opale, 259 p.

EL MIMOUNI A., DAOUDI L., SAIDI M.E., BAIDDAH A. (2010). Comportement hydrologique et dynamique d'un bassin versant en milieu semi-aride: exemple du bassin versant du Ksob (HAUT atlas occidental, Maroc). Revue Quaternario y Geomorphologia, Vol. 24 (1-2), pp 99-112.

FOLK R.L, WARD W.C. (1957). Barazos river bar : a stadyin the significane of grain size parameters. Journal of Sedimentary Petrology, Vol. 27 (1), pp 3-26.

GENTILE W. (1997). Caractérisation et suivi d'un champ dunaire par analyses sédimentologiques et télédétection (Essaouira-Cap Sim, Maroc Atlantique). Thèse Université de Province - Aix-Marseille I, 307 p.

HANDER M. (1993). Contribution à l'étude de la bioclimatologie humaine au Maroc: l'exemple d'Essaouira. Thèse de Doctorat, Paris-IV, 356 p. 
LHARTI S., FLOR G., DAOUDI L., FLOR G.B., EL MIMOUNI A., BEN ALI A. (2006). Morfologia y Sedimentologia del complejo playa/dunas costeras de Essaouira (Marruecos atlantico) : modelo de transporte costero. Actas de la IX Reunión Nacional de Geomorfología, Santiago de Compostela (A. Pérez Alberti y J. López Bedoya, ed.), pp 401-417.

REICHMÜTH B. (2003). Contribution à la connaissance de la morphodynamique des plages à barres intertidales, approche expérimentale. Côte d’Opale, Nord de la France. Thèse de doctorat, Université du Littoral Côte d'Opale, Dunkerque, 248 p.

REICHMÜTH B., ANTHONY E.J. (2007). Tidal influence on the intertidal bar morphology of two contrasting macrotidal beaches. Geomorphology, Vol. 90, Issues 12, pp 101-114. doi:10.1016/j.geomorph.2007.01.015

RIVIERE A. (1977). Méthodes granulométriques - Techniques et Interprétation. Masson, Paris, 170 p.

SEDRATI M. (2006). Morphodynamique et transport hydro-sédimentaire sur les estrans macrotidaux du Nor-Pas-de-Calais : Implications en aménagement du littoral. Thèse de doctorat l'Université du Littoral Côte d'Opale, 215 p.

SEDRATI M., ANTHONY E.J. (2007). Storm-generated morphological change and longshore sand transport in the intertidal zone of a multi-barred macrotidal beach. Marine Geology, Vol. 244, Issues 1-4, pp 209-229. doi:10.1016/j.margeo.2007.07.002

SIMON C. (2000). Le géo-système dunaire anthropisé d'Essaouira - est (Maroc Atlantique) dynamique et paléo-environnements. Thèse de doctorat. Univ. AixMarseille I, 204 p.

WEISROCK A. (1980). Géomorphologie et paléo-environnements de l'Atlas atlantique (Maroc). Thèse d'état, Paris I, 931 p. 